Bull. Fac .Agric., Cairo Univ. 64:129-139 (2013).

\title{
EVALUATION OF BOLL COMPONENT EFFECTS ON COTTON YIELD
}

\author{
(Received:9.6.2013)
}

\author{
By \\ H. A. Idris and H. B. Abou Tour \\ Cotton Research Institute, Agricultural Research Center, Giza, Egypt
}

\begin{abstract}
The present investigation deals with the classification of the effects of boll components on yield for some Egyptian cotton genotypes (Gossypium barbadense L.) viz., G.80, G90, (G83 x (G75 x 5844)) x G80 and G90 x Australian. These Genotypes were evaluated in three locations in the Upper Egypt (Beni Souif, Minya and Assuit) during three seasons (2009, 2010 and 2011), except 2010 season for Assuit. A randomized complete block design with four replications was used. Two samples (50 bolls each) were obtained from each plot in each location during the three seasons. Genotypes were evaluated for yield (seed cotton per boll) and boll components (dry weight, lint cotton weight, seeds weight and number of seeds per boll). The analysis of variance of samples revealed significant differences among genotypes with respect to dry weight per boll and number of seeds per boll. G80 and G90 significantly surpassed the other genotypes with respect to dry weight per boll and number of seeds per boll, respectively. (G83 x (G75 x 5844)) x G80 was the best genotype, showing the lowest values of variance for yield and boll components under different locations indicating that its performance was slightly affected by locations. The boll components were classified into two groups. The first group includes dry weight and lint cotton weight. The second group includes seeds weight and number of seeds. Estimates of simple, partial and multiple correlation coefficients between yield and boll components were calculated. The results of the first group exhibited that dry weight alone accounted for $45.7 \%, 29.9 \%, 22.3 \%$ and $3 \%$ of the variability in yield of G80, G90, (G83 x (G75 x 5844)) x G80 and G90 x Australian, respectively. Lint cotton weight alone accounted for $94.3 \%, 92.5 \%, 90.9 \%$ and $95.3 \%$ of the variability in yield of genotypes in the same above mentioned order. Both dry and lint weight per boll jointly accounted for $94.4 \%, 92.7 \%, 91 \%$ and $95.3 \%$ of the variability in yield of the same order of genotypes. The results of the second group revealed that seeds weight per boll alone accounted for $98 \%, 96.8 \%, 96.3 \%$ and $98 \%$ of the variability in yield of G80, G90, (G83 x (G75 x 5844)) x G80 and G90 x Australian, respectively. The number of seeds per boll alone accounted for $43.4 \%, 44 \%, 34.6 \%$ and $45.1 \%$ of the variability in yield of genotypes in the same order. Both seeds weight and the number of seeds per boll jointly accounted for $98.3 \%, 97.6 \%, 97.4 \%$ and $98.4 \%$ of the variability in yield of genotypes in the same above mentioned order of genotypes. The present study is very important for the breeder and regional programs with respect to the objective and statistical analysis.
\end{abstract}

Key words: Boll components, cotton yield, genotypes, locations, samples analysis.

\section{INTRODUCTION}

Developing high yielding cotton cultivars is considered the main objective of any plant breeding program. Seed cotton yield is a complex quantitative character greatly affected by many environmental factors. Selection based on yield itself is often not effective because of the confounding effects of the environment. Knowledge of the magnitude and type of the interrelationships between characters has important practical implications in plant breeding. For this reason, plant breeding pay much attention to study the association among different traits. Another approach towards improvement of yield may be to emphasize selection for its components. However, it is important to examine the contribution of each of the various components in order to give more attention to those having the greater influence on yield. Studying the correlation among different economic characters of cotton is of great interest to the plant breeder. Correlation simply measures the apparent mutual association between the two variables regardless of the cause (Idris ,2002). 
Mahrous et al. (2012) noticed that correlation coefficient expresses the magnitude of relationship between various plant characters and determine the component character on which selection can be based for improvement of seed cotton yield. The true picture of correlation between seed cotton yield and traits is reflected from the direct effect of that trait which will help identifying the trait that contribute directly to improve seed cotton yield.

The correlation between two variables, disregarding any other variables that may be varying simultaneously, is called simple correlation. The correlation between two variables, when one or more other variables are held at a constant level, is called partial correlation. The combined relation between a variable and two or more other variables varying simultaneously is called multiple correlation (Little and Hills, 1978).

Patil and Mensinkai (1972) noted that positive and significant correlation coefficient was found between seed cotton yield per plant and boll weight. Gill (1981) investigated eight characters in 62 diverse $G$. hirsutum strains in four environments. They indicated that boll size has important positive direct effects on seed cotton yield.

The objective of the present study was to estimate the effects of boll components on cotton yield.

\section{MATERIALS AND METHODS}

Four Egyptian cotton genotypes (Gossypium barbadense L.) were evaluated at three locations in Upper Egypt (Beni Souif, Minya and Assuit) during three seasons (2009, 2010 and 2011), except 2010 season for Assuit. Two of the genotypes were cultivars, viz. G.80 and G90. The two remaining genotypes were hybrids, viz. (G83 x (G75 x 5844)) x G80 and G90 x Australian. A randomized complete block design with four replications was used. Two samples were obtained from each plot in individual locations during the three seasons (Table 1). Planting was during the last week of March. All agricultural practices were done as recommended.

Genotypes were evaluated for yield (seed cotton per boll) and boll components (dry weight, lint cotton weight, seeds weight and number of seeds).

\subsection{Statistical analysis 2.1.1.Samples Analysis}

The analysis of variance of samples is illustrated in Table (2).

Statistical analysis of individual and all locations followed Fowler et al. (1998). The means were compared by Tukey test as given by the same author. All comparisons were done at 0.05 level of significance.

\subsubsection{Correlation coefficients}

The boll components were classified into two groups. The first group includes dry weight $\left(\mathrm{x}_{1}\right)$ and lint cotton weight $\left(\mathrm{x}_{2}\right)$. The second group includes seeds weight $\left(\mathrm{x}_{1}\right)$ and number of seeds $\left(\mathrm{x}_{2}\right)$. Statistical analysis of simple, partial and multiple correlations between seed cotton per boll (y) and boll components (x) was straightforward as shown by Little and Hills (1978) and Roger (1994).

\section{RESULTS AND DISCUSSION 3.1 Samples Analysis 3.1.1 Individual locations}

The analysis of variance for individual locations during the three seasons, with respect to seed cotton yield per boll and boll components (dry weight, lint cotton weight, seeds weight and number of seeds)revealed the presence of significant differences among genotypes(Table 3).

Significant variation due to genotypes was observed for dry weight per boll and number of seeds per boll in the three locations. In contrast, non-significant variation due to genotypes was detected for yield (seed cotton per boll), lint cotton weight per boll and seeds weight per boll in the three locations except for lint cotton per boll in Minia.

G80 had the highest mean for dry weight per boll in the three locations. It significantly exceeded all other genotypes except the two new genotypes (G83 x (G75 x 5844)) x G80 and G90 x Australian in Assuit. G80 gave the same results in Minia with respect to lint cotton weight per boll, as it did not differ significantly from the two new genotypes (Table 4).

G90 x Australian had the highest mean for the number of seeds per boll at the three locations. It significantly surpassed the other genotypes except G90 at Assuit location and (G83 x (G75 x 5844)) x G80 at Minia and Assuit locations, (Table 4).

The analysis of variance showed that (G83 x (G75 x 5844)) x G80 was the best genotype at the three locations. It gave the lowest values of variance for yield and boll components compared to other genotypes, except for number of seeds per 
Table (1): Number of samples and sample size for individual genotypes

\begin{tabular}{|c|c|c|c|c|}
\hline & \multicolumn{3}{|c|}{ Individual location } & \multirow[t]{2}{*}{ Both locations } \\
\hline Season & Beni Souif & Minia & Assuit & \\
\hline 2009 & $\begin{array}{l}\text { Number }=8 \\
\text { Size }=50 \text { bolls }\end{array}$ & $\begin{array}{l}\text { Number }=8 \\
\text { Size }=50 \text { bolls }\end{array}$ & $\begin{array}{l}\text { Number }=8 \\
\text { Size }=50 \text { bolls }\end{array}$ & \\
\hline 2010 & $\begin{array}{l}\text { Number }=8 \\
\text { Size }=50 \text { bolls }\end{array}$ & $\begin{array}{l}\text { Number }=8 \\
\text { Size }=50 \text { bolls }\end{array}$ & -------- & \\
\hline 2011 & $\begin{array}{l}\text { Number }=8 \\
\text { Size }=50 \text { bolls }\end{array}$ & $\begin{array}{l}\text { Number }=8 \\
\text { Size }=50 \text { bolls }\end{array}$ & $\begin{array}{l}\text { Number }=8 \\
\text { Size }=50 \text { bolls }\end{array}$ & \\
\hline Total & Number $=24$ & Number $=24$ & Number $=16$ & Number $=64$ \\
\hline
\end{tabular}

Table (2):One - way ANOVA of classification of boll.

\begin{tabular}{|l|c|}
\hline \multicolumn{1}{|c|}{ Source of variation } & df \\
\hline $\begin{array}{l}\text { Among genotypes } \\
\text { Within genotypes }\end{array}$ & $\begin{array}{c}\mathrm{g}-1 \\
\left(\mathrm{n}_{\mathrm{T}}-\mathrm{g}\right)\end{array}$ \\
\hline Total & $\mathrm{n}_{\mathrm{T}}-1$ \\
\hline
\end{tabular}

Where: $\mathrm{g}$ = Number of genotypes

$\mathrm{n}_{\mathrm{T}}=$ Number of total samples

boll at both Minia and Assuit. The results show that the new genotype was slightly affected by seasonal variation within individual locations (Table 3).

\subsubsection{Analysis over locations}

The analysis of variance over both locations during the three seasons, with respect to yield (seed cotton per boll) and boll components (dry weight, lint cotton weight, seeds weight and number of seeds) revealed the presence of significant differences among genotypes (Table $3)$.

The genotypes exhibited significant differences with respect to dry weight per boll and number of seeds per boll. G80 significantly surpassed the other genotypes with respect to dry weight per boll. G90 x Australian significantly exceeded the other genotypes for number of seeds per boll (Table 4).

On the other hand, the analysis of variance showed that the new genotype (G83 x (G75 x 5844)) x G80 was the best genotype. It showed the lowest variance for yield and boll components compared to other genotypes at different locations. The results show that the performance of this hybrid was slightly affected by locations (more stable).

The above results are in line with the finding of Idris et al. (2011). They evaluated three Egyptian cotton genotypes in the Upper Egypt. Analysis of variance over locations showed that (G83 x (G75 $x$ 5844)) $x$ G80 showed the lowest variance between locations for seed cotton per boll, dry weight per boll and number of seeds per boll.

\subsection{Correlation between traits}

The results in Table (5) show the analysis of simple, partial and multiple correlations between seed cotton yield per boll (y) and two groups of boll components. The first group includes dry weight per boll $\left(\mathrm{x}_{1}\right)$ and lint cotton per boll $\left(\mathrm{x}_{2}\right)$. The second group includes seeds weight per boll $\left(\mathrm{x}_{1}\right)$ and the number of seeds per boll $\left(\mathrm{x}_{2}\right)$.

\subsubsection{Individual locations}

Concerning the first group, at Beni Souf, dry weight and lint weight per boll were significantly positively simply correlated with yield for all genotypes. In Minia and Assuit, lint weight was significantly positively simply correlated with yield for all genotypes except G80 in Minia. On the contrary, at the same two locations, dry weight per boll showed non-significant positive simple correlation with yield for all genotypes except G80 in Assuit.

At the three locations, dry weight per boll was non-significantly positively partially correlated with yield when lint weight per boll is held constant for all genotypes. In contrast, at Beni Souif and Assuit, lint weight per boll was significantly positively partially correlated with yield when dry weight per boll is held constant for all genotypes.

At the three locations, both dry weight and lint weight per boll showed significant positive multiple correlation with yield for all genotypes except G80 and G90 in Minia.

Concerning the second group, in Beni Souf and Assuit, seeds weight and the number of seeds per boll showed significant positive simple correlation with yield for all genotypes except (G83 x (G75 x 5844)) x G80 at Assuit. In Minia, seeds weight per boll was significantly positively simply correlated with yield for all genotypes except G80. In contrast, the number of seeds per boll was non-significantly positively simply correlated with yield for all genotypes in the same location. 
Table (3): Mean squares of location effects on cotton genotypes.

\begin{tabular}{|c|c|c|c|c|c|c|c|}
\hline \multirow[b]{3}{*}{ Source of variation } & \multicolumn{7}{|c|}{ Yield (g) (seed cotton per boll) } \\
\hline & \multicolumn{5}{|c|}{ Individual locations } & \multicolumn{2}{|c|}{ Both locations } \\
\hline & df & Beni Souif & Minia & df & Assuit & df & Locations \\
\hline Among genotypes & 3 & 0.043 & 0.113 & 3 & 0.020 & 3 & 0.011 \\
\hline Within genotypes & 92 & 0.073 & 0.060 & 60 & 0.099 & 252 & 0.085 \\
\hline 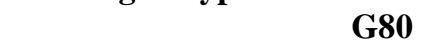 & 23 & 0.106 & 0.066 & 15 & 0.098 & 63 & 0.110 \\
\hline G90 & 23 & 0.071 & 0.063 & 15 & 0.080 & 63 & 0.071 \\
\hline$(G 83 \times(G 75 \times 5844)) \times G 80$ & 23 & 0.055 & 0.053 & 15 & 0.042 & 63 & 0.064 \\
\hline G90 x Australian & 23 & 0.060 & 0.059 & 15 & 0.174 & 63 & 0.094 \\
\hline Total & 95 & & & 63 & & 255 & \\
\hline \multirow[b]{2}{*}{ Source of variation } & \multicolumn{7}{|c|}{ Dry weight per boll (g) } \\
\hline & df & Beni Souif & Minia & df & Assuit & df & Locations \\
\hline Among genotypes & 3 & $0.109 * *$ & $0.265 * *$ & 3 & $0.091 * *$ & 3 & $0.423 * *$ \\
\hline Within genotypes & 92 & 0.014 & 0.012 & 60 & 0.019 & 252 & 0.015 \\
\hline G80 & 23 & 0.022 & 0.020 & 15 & 0.044 & 63 & 0.028 \\
\hline G90 & 23 & 0.020 & 0.009 & 15 & 0.009 & 63 & 0.013 \\
\hline$(G 83 \times(G 75 \times 5844)) \times$ G80 & 23 & 0.005 & 0.005 & 15 & 0.004 & 63 & 0.006 \\
\hline G90 x Australian & 23 & 0.008 & 0.014 & 15 & 0.021 & 63 & 0.013 \\
\hline Total & 95 & & & 63 & & 255 & \\
\hline \multirow[b]{2}{*}{ Source of variation } & \multicolumn{7}{|c|}{ lint cotton weight per boll (g) } \\
\hline & df & Beni Souif & Minia & df & Assuit & df & Locations \\
\hline Among genotypes & 3 & 0.007 & $0.044 * *$ & 3 & 0.001 & 3 & 0.023 \\
\hline Within genotypes & 92 & 0.012 & 0.010 & 60 & 0.016 & 252 & 0.013 \\
\hline G80 & 23 & 0.017 & 0.011 & 15 & 0.015 & 63 & 0.016 \\
\hline G90 & 23 & 0.011 & 0.011 & 15 & 0.013 & 63 & 0.011 \\
\hline$(G 83 \times(G 75 \times 5844)) \times$ G80 & 23 & 0.010 & 0.010 & 15 & 0.007 & 63 & 0.011 \\
\hline G90 x Australian & 23 & 0.010 & 0.010 & 15 & 0.028 & 63 & 0.015 \\
\hline Total & 95 & & & 63 & & 255 & \\
\hline \multirow[b]{2}{*}{ Source of variation } & \multicolumn{7}{|c|}{ Seeds weight per boll (g) } \\
\hline & df & Beni Souif & Minia & df & Assuit & df & Locations \\
\hline Among genotypes & 3 & 0.035 & 0.022 & 3 & 0.023 & 3 & 0.011 \\
\hline Within genotypes & 92 & 0.027 & 0.024 & 60 & 0.037 & 252 & 0.033 \\
\hline G80 & 23 & 0.040 & 0.027 & 15 & 0.037 & 63 & 0.045 \\
\hline G90 & 23 & 0.027 & 0.024 & 15 & 0.033 & 63 & 0.028 \\
\hline$($ G83 x $($ G75 x 5844) $) \times$ G80 & 23 & 0.019 & 0.021 & 15 & 0.015 & 63 & 0.025 \\
\hline G90 x Australian & 23 & 0.023 & 0.023 & 15 & 0.064 & 63 & 0.036 \\
\hline Total & 95 & & & 63 & & 255 & \\
\hline \multirow[b]{2}{*}{ Source of variation } & \multicolumn{7}{|c|}{ Number of seeds per boll } \\
\hline & df & Beni Souif & Minia & df & Assuit & df & Locations \\
\hline $\begin{array}{l}\text { Among genotypes } \\
\text { Within genotypes }\end{array}$ & $\begin{array}{l}3 \\
92\end{array}$ & $\begin{array}{l}49.95 * * \\
3.67\end{array}$ & $\begin{array}{l}16.19 * * \\
2.46\end{array}$ & $\begin{array}{l}3 \\
60\end{array}$ & $\begin{array}{l}8.07 * \\
2.50\end{array}$ & $\begin{array}{l}3 \\
252\end{array}$ & $\begin{array}{l}64.52 * * \\
2.95\end{array}$ \\
\hline G80 & 23 & 5.27 & 1.57 & 15 & 1.72 & 63 & 2.98 \\
\hline G90 & 23 & 3.32 & 3.18 & 15 & 2.51 & 63 & 3.06 \\
\hline$($ G83 $\times($ G75 $\times 5844)) \times$ G80 & 23 & 1.91 & 2.85 & 15 & 2.51 & 63 & 2.36 \\
\hline G90 x Australian & 23 & 4.16 & 2.24 & 15 & 3.26 & 63 & 3.40 \\
\hline Total & 95 & & & 63 & & 255 & \\
\hline
\end{tabular}


Table (4): Mean squares of location effects on cotton genotypes.

\begin{tabular}{|c|c|c|c|c|}
\hline \multirow[b]{3}{*}{ Genotypes } & \multicolumn{4}{|c|}{ Yield (g) (seed cotton per boll) } \\
\hline & \multicolumn{3}{|c|}{ Individual locations } & \multirow[t]{2}{*}{ Both locations } \\
\hline & Beni Souif & Minia & Assuit & \\
\hline G80 & 2.45 & 2.70 & 2.34 & 2.52 \\
\hline G90 & 2.52 & 2.54 & 2.42 & 2.51 \\
\hline$(G 83 \times(G 75 \times 5844)) \times$ G80 & 2.47 & 2.65 & 2.36 & 2.51 \\
\hline G90 x Australian & 2.54 & 2.63 & 2.39 & 2.54 \\
\hline Tukey & \multirow{2}{*}{\multicolumn{4}{|c|}{ Dry weight per boll (g) }} \\
\hline \multirow[b]{2}{*}{ Genotypes } & & & & \\
\hline & Beni Souif & Minia & Assuit & locations \\
\hline G80 & 1.07 & 1.18 & 1.09 & 1.11 \\
\hline G90 & 0.92 & 0.95 & 0.90 & 0.92 \\
\hline$(G 83 \times(G 75 \times 5844)) \times$ G80 & 0.97 & 1.03 & 1.02 & 1.00 \\
\hline G90 x Australian & 0.94 & 0.96 & 1.01 & 0.97 \\
\hline Tukey & 0.09 & 0.08 & 0.13 & 0.06 \\
\hline \multirow[b]{2}{*}{ Genotypes } & \multicolumn{4}{|c|}{ lint cotton weight per boll (g) } \\
\hline & Beni Souif & Minia & Assuit & locations \\
\hline G80 & 1.02 & 1.08 & 0.96 & 1.03 \\
\hline G90 & 1.01 & 0.98 & 0.97 & 0.99 \\
\hline$(G 83 \times(G 75 \times 5844)) \times$ G80 & 1.01 & 1.07 & 0.96 & 1.02 \\
\hline G90 x Australian & 1.05 & 1.06 & 0.97 & 1.03 \\
\hline Tukey & -- & 0.08 & -- & -- \\
\hline \multirow[b]{2}{*}{ Genotypes } & \multicolumn{4}{|c|}{ Seeds weight per boll (g) } \\
\hline & Beni Souif & Minia & Assuit & locations \\
\hline G80 & 1.43 & 1.63 & 1.38 & 1.49 \\
\hline G90 & 1.51 & 1.55 & 1.47 & 1.52 \\
\hline$(G 83 \times(G 75 \times 5844)) \times$ G80 & 1.46 & 1.59 & 1.39 & 1.49 \\
\hline G90 x Australian & 1.49 & 1.57 & 1.41 & 1.50 \\
\hline Tukey & -- & -- & -- & -- \\
\hline \multirow[b]{2}{*}{ Genotypes } & \multicolumn{4}{|c|}{ Number of seeds per boll } \\
\hline & Beni Souif & Minia & Assuit & locations \\
\hline G80 & 15.03 & 15.56 & 15.57 & 15.36 \\
\hline G90 & 16.25 & 15.92 & 16.69 & 16.23 \\
\hline$(G 83 \times(G 75 \times 5844)) \times$ G80 & 15.96 & 16.33 & 16.15 & 16.15 \\
\hline G90 x Australian & 18.43 & 17.45 & 17.22 & 17.76 \\
\hline Tukey & 1.45 & 1.19 & 1.48 & 0.78 \\
\hline
\end{tabular}

--: Not significant at .05 level.

In Beni Souif and Assuit, seeds weight per boll was significantly positively partially correlated with yield when the number of seeds per boll is held constant for all genotypes. On the contrary, at the three locations, the number of seeds per boll showed non-significant positive partial correlation with yield when seeds weight per boll is held constant for all genotypes except G90 x Australian at Beni Souif.

In the three locations, both seeds weight and the number of seeds per boll showed significant positive multiple correlation with yield for all genotypes except G80 in Minia.

\subsubsection{Over locations}

Concerning the first group, dry weight and lint weight per boll were significantly positively simply correlated with yield for all genotypes except (G90 x Australian), where the dry weight per boll was non-significantly correlated with yield.

Dry weight per boll showed non-significant positive partially correlation with yield when lint weight per boll is held constant for all genotypes. In contrast, lint weight per boll was significantly positive particle correlated with yield when dry weight per boll is held constant for all genotypes.

Both dry and lint weight per boll showed significant positive multiple correlation with yield for all genotypes.

The results of the first group of boll components show that dry weight per boll alone accounted for $45.7 \%, 29.9 \%, 22.3 \%$ and $3 \%$ of 
Table (5): Correlations among seed cotton per boll (y) and two groups of boll components (x).

\begin{tabular}{|c|c|c|c|c|c|c|}
\hline \multicolumn{7}{|c|}{ G80 } \\
\hline \multirow[b]{3}{*}{ Correlations } & \multicolumn{6}{|c|}{ First group in individual locations } \\
\hline & \multicolumn{2}{|c|}{ Beni Souif } & \multicolumn{2}{|c|}{ Minia } & \multicolumn{2}{|c|}{ Assuit } \\
\hline & \multirow{2}{*}{$\begin{array}{c}\text { Dry weight } \\
\left(\mathbf{x}_{1}\right)\end{array}$} & \multirow{2}{*}{$\begin{array}{c}\text { Lint weight } \\
\left(\mathrm{x}_{2}\right)\end{array}$} & \multirow{2}{*}{$\begin{array}{c}\text { Dry weight } \\
\left(\mathbf{x}_{1}\right) \\
\end{array}$} & \multirow{2}{*}{$\begin{array}{c}\text { Lint weight } \\
\left(\mathbf{x}_{2}\right) \\
\end{array}$} & \multirow{2}{*}{$\begin{array}{c}\text { Dry weight } \\
\left(\mathbf{x}_{1}\right)\end{array}$} & \multirow{2}{*}{$\begin{array}{c}\text { Lint weight } \\
\left(\mathbf{x}_{2}\right)\end{array}$} \\
\hline Simple & & & & & & \\
\hline $\mathbf{r}^{2} \mathbf{y} x$ & 0.455 & 0.982 & 0.036 & 0.130 & 0.684 & 0.991 \\
\hline $\mathbf{r} \mathbf{y} x$ & $0.675^{* *}$ & $0.991 * *$ & 0.190 & 0.360 & $0.827 * *$ & $0.995 * *$ \\
\hline $\mathbf{r}^{2} \mathbf{x}_{1} \mathbf{x}_{2}$ & \multirow{2}{*}{\multicolumn{2}{|c|}{$\begin{array}{l}0.451 \\
0.671 * *\end{array}$}} & \multirow{2}{*}{\multicolumn{2}{|c|}{$\begin{array}{l}0.041 \\
0.203 \\
\end{array}$}} & \multirow{2}{*}{\multicolumn{2}{|c|}{$\begin{array}{l}0.684 \\
0.827 * *\end{array}$}} \\
\hline $\mathbf{r} \mathbf{x}_{1} \mathbf{x}_{2}$ & & & & & & \\
\hline Partial & $\left(\mathbf{x}_{1}\right)$ & $\left(\mathbf{x}_{2}\right)$ & $\left(\mathbf{x}_{1}\right)$ & $\left(\mathbf{x}_{2}\right)$ & $\left(\mathbf{x}_{1}\right)$ & $\left(\mathbf{x}_{2}\right)$ \\
\hline $\mathbf{r}^{2} \mathbf{y} \mathbf{x}_{1} \cdot \mathbf{x}_{2}$ & \multirow{4}{*}{$\begin{array}{l}0.009 \\
0.093\end{array}$} & & \multirow{4}{*}{$\begin{array}{l}0.016 \\
0.128\end{array}$} & & 0.005 & \\
\hline $\mathbf{r} \mathbf{y} \mathbf{x}_{1} \cdot \mathbf{x}_{2}$ & & & & & \multirow[t]{3}{*}{0.070} & \\
\hline $\mathbf{r}^{2} \mathbf{y} x_{2} \cdot x_{1}$ & & 0.967 & & 0.112 & & 0.971 \\
\hline $\mathbf{r} \mathbf{y} \mathbf{x}_{2} \cdot \mathbf{x}_{1}$ & & $0.983 * *$ & & 0.334 & & $0.985 * *$ \\
\hline Multiple & $\left(\mathbf{x}_{1}\right)$ & $\left(\mathbf{x}_{2}\right)$ & $\left(\mathbf{x}_{1}\right)$ & $\left(\mathbf{x}_{2}\right)$ & $\left(\mathbf{x}_{1}\right)$ & $\left(\mathbf{x}_{2}\right)$ \\
\hline$R^{2} \mathbf{y} \cdot \mathbf{x}_{1} \mathbf{x}_{2}$ & \multirow{2}{*}{\multicolumn{2}{|c|}{$\begin{array}{l}0.982 \\
0.991 * *\end{array}$}} & \multirow{2}{*}{\multicolumn{2}{|c|}{$\begin{array}{l}1 \\
0.144 \\
0.379 \\
\end{array}$}} & \multirow{2}{*}{\multicolumn{2}{|c|}{$\begin{array}{l}0.991 \\
0.995^{* *}\end{array}$}} \\
\hline$R y . x_{1} x_{2}$ & & & & & & \\
\hline & & Second g & oup in individ & I locations & & \\
\hline & Beni & ouif & & & & \\
\hline Correlations & Seeds weight & No. Seeds & Seeds weight & No. Seeds & Seeds weight & No. Seeds \\
\hline Simple & $\left(\mathbf{x}_{1}\right)$ & $\left(\mathbf{x}_{2}\right)$ & $\left(\mathbf{x}_{1}\right)$ & $\left(\mathbf{x}_{2}\right)$ & $\left(\mathbf{x}_{1}\right)$ & $\left(\mathbf{x}_{2}\right)$ \\
\hline $\mathbf{r}^{2} \mathbf{y} x$ & 0.991 & 0.839 & 0.148 & 0.016 & 0.996 & 0.260 \\
\hline $\mathbf{r y x}$ & $0.996 * *$ & $0.916 * *$ & 0.385 & 0.127 & $0.998 * *$ & $0.510 *$ \\
\hline $\mathbf{r}^{2} \mathbf{x}_{1} \mathbf{x}_{2}$ & & & & & & \\
\hline$r \mathbf{x}_{1} \mathbf{x}_{2}$ & 0.5 & $4 * *$ & & & & \\
\hline Partial & $\left(\mathbf{x}_{1}\right)$ & $\left(\mathbf{x}_{2}\right)$ & $\left(\mathbf{x}_{1}\right)$ & $\left(\mathbf{x}_{2}\right)$ & $\left(\mathbf{x}_{1}\right)$ & $\left(\mathbf{x}_{2}\right)$ \\
\hline $\mathbf{r}^{2} \mathbf{y} x_{1} \cdot x_{2}$ & 0.955 & & 0.142 & & 0.996 & \\
\hline $\mathbf{r y x} x_{1} \cdot x_{2}$ & $0.977 * *$ & & 0.377 & & $0.998 * *$ & \\
\hline $\mathbf{r}^{2} \mathbf{y} x_{2} \cdot x_{1}$ & & 0.162 & & 0.009 & & 0.192 \\
\hline $\mathbf{r} \mathrm{yx}_{2} \cdot \mathrm{x}_{1}$ & & 0.402 & & 0.096 & & 0.438 \\
\hline Multiple & $\left(\mathbf{x}_{1}\right)$ & $\left(\mathbf{x}_{2}\right)$ & $\left(\mathbf{x}_{1}\right)$ & $\left(\mathbf{x}_{2}\right)$ & $\left(\mathbf{x}_{1}\right)$ & $\left(\mathbf{x}_{2}\right)$ \\
\hline$R^{2} y \cdot x_{1} x_{2}$ & 0.9 & & & & & \\
\hline$R y \cdot x_{1} x_{2}$ & 0.5 & $6 * *$ & & & & $8 * *$ \\
\hline & & Two & roups in both & cations & & \\
\hline Correlations & Dry weight & Lint weight & & Seeds weight & No. Seeds & \\
\hline Simple & $\left(\mathbf{x}_{1}\right)$ & $\left(\mathbf{x}_{2}\right)$ & & $\left(\mathbf{x}_{1}\right)$ & $\left(\mathbf{x}_{2}\right)$ & \\
\hline $\mathbf{r}^{2} \mathbf{y x}$ & 0.457 & 0.943 & & 0.980 & 0.434 & \\
\hline $\mathbf{r} \mathbf{y} x$ & $0.676^{* *}$ & $0.971 * *$ & & $0.990 * *$ & $0.658 * *$ & \\
\hline $\mathbf{r}^{2} \mathbf{x}_{1} \mathbf{x}_{2}$ & 0. & & & & & \\
\hline $\mathbf{r} \mathbf{x}_{1} \mathbf{x}_{2}$ & & $0 * *$ & & & $19 * *$ & \\
\hline Partial & $\left(\mathbf{x}_{1}\right)$ & $\left(\mathbf{x}_{2}\right)$ & & $\left(\mathbf{x}_{1}\right)$ & $\left(\mathbf{x}_{2}\right)$ & \\
\hline $\mathbf{r}^{2} \mathbf{y} x_{1} \cdot x_{2}$ & 0.021 & & & 0.970 & & \\
\hline $\mathbf{r y x _ { 1 }} \cdot \mathrm{x}_{2}$ & 0.146 & & & $0.985 * *$ & & \\
\hline $\mathbf{r}^{2} \mathbf{y} \mathbf{x}_{2} \cdot \mathbf{x}_{1}$ & & 0.897 & & & 0.165 & \\
\hline $\mathbf{r} \mathbf{y} x_{2} \cdot x_{1}$ & & $0.947 * *$ & & & $0.406 * *$ & \\
\hline Multiple & $\left(\mathbf{x}_{1}\right)$ & $\left(\mathbf{x}_{2}\right)$ & & $\left(\mathbf{x}_{1}\right)$ & $\left(\mathbf{x}_{2}\right)$ & \\
\hline $\begin{array}{l}R^{2} y \cdot x_{1} x_{2} \\
R y \cdot x_{1} x_{2}\end{array}$ & $\begin{array}{l}0.5 \\
0.5\end{array}$ & & & & $33 *$ & \\
\hline
\end{tabular}


Table (5): Cont. I

\begin{tabular}{|c|c|c|c|c|c|c|}
\hline \multicolumn{7}{|c|}{ G90 } \\
\hline \multirow[b]{3}{*}{ Correlations } & \multicolumn{6}{|c|}{ First group in individual locations } \\
\hline & \multicolumn{2}{|c|}{ Beni Souif } & \multicolumn{2}{|c|}{ Minia } & \multicolumn{2}{|c|}{ Assuit } \\
\hline & \multirow{2}{*}{$\begin{array}{c}\text { Dry weight } \\
\left(\mathrm{x}_{1}\right)\end{array}$} & \multirow{2}{*}{$\begin{array}{l}\text { Lint weight } \\
\left(\mathbf{x}_{2}\right)\end{array}$} & \multirow{2}{*}{$\begin{array}{c}\text { Dry weight } \\
\left(\mathrm{x}_{1}\right)\end{array}$} & \multirow{2}{*}{$\begin{array}{c}\text { Lint weight } \\
\left(\mathbf{x}_{2}\right)\end{array}$} & \multirow{2}{*}{ Dry weight } & \multirow{2}{*}{$\begin{array}{c}\text { Lint weight } \\
\left(x_{2}\right)\end{array}$} \\
\hline Simple & & & & & & \\
\hline $\mathbf{r}^{2} \mathbf{y} x$ & 0.627 & 0.942 & 0.032 & 0.212 & 0.020 & 0.987 \\
\hline $\mathbf{r} \mathbf{y} \mathbf{x}$ & $0.792 * *$ & $0.971 * *$ & 0.179 & $0.461 *$ & 0.143 & $0.993 * *$ \\
\hline $\mathbf{r}^{2} \mathbf{x}_{1} \mathbf{x}_{2}$ & \multicolumn{2}{|c|}{0.592} & \multicolumn{2}{|c|}{0.041} & \multicolumn{2}{|c|}{0.035} \\
\hline $\mathbf{r} \mathbf{x}_{1} \mathbf{x}_{2}$ & \multicolumn{2}{|c|}{$0.770 * *$} & \multicolumn{2}{|c|}{0.201} & \multicolumn{2}{|c|}{0.187} \\
\hline Partial & $\left(\mathbf{x}_{1}\right)$ & $\left(\mathbf{x}_{2}\right)$ & $\left(\mathbf{x}_{1}\right)$ & $\left(\mathbf{x}_{2}\right)$ & $\left(\mathbf{x}_{1}\right)$ & $\left(\mathbf{x}_{2}\right)$ \\
\hline $\mathbf{r}^{2} \mathbf{y} \mathbf{x}_{1} \cdot \mathbf{x}_{2}$ & \multirow{4}{*}{$\begin{array}{l}0.086 \\
0.292\end{array}$} & \multirow{4}{*}{$\begin{array}{l}0.858 \\
0.926^{* *}\end{array}$} & \multirow{4}{*}{$\begin{array}{l}0.010 \\
0.099\end{array}$} & & 0.144 & \\
\hline $\mathbf{r} \mathbf{y} \mathbf{x}_{1} \cdot \mathbf{x}_{2}$ & & & & & 0.380 & \\
\hline $\mathbf{r}^{2} \mathbf{y} \mathbf{x}_{2} \cdot \mathbf{x}_{1}$ & & & & 0.194 & & 0.988 \\
\hline $\mathbf{r} \mathbf{y} \mathbf{x}_{2} \cdot \mathbf{x}_{1}$ & & & & 0.441 & & $0.994 * *$ \\
\hline Multiple & $\left(\mathbf{x}_{1}\right)$ & $\left(\mathbf{x}_{2}\right)$ & $\left(\mathbf{x}_{1}\right)$ & $\left(\mathbf{x}_{2}\right)$ & $\left(\mathbf{x}_{1}\right)$ & $\left(\mathbf{x}_{2}\right)$ \\
\hline$R^{2} y \cdot x_{1} x_{2}$ & & & & & & \\
\hline$R y \cdot x_{1} x_{2}$ & & $73 * *$ & & & & $* *$ \\
\hline & & Secon & roup in indiv & locations & & \\
\hline & Beni & ouif & & & & \\
\hline Correlations & Seeds & No. Seeds & Seeds weight & No. Seeds & Seeds weight & No. Seeds \\
\hline Simple & $\left(\mathbf{x}_{1}\right)$ & $\left(\mathbf{x}_{2}\right)$ & $\left(\mathbf{x}_{1}\right)$ & $\left(\mathbf{x}_{2}\right)$ & $\left(\mathbf{x}_{1}\right)$ & $\left(\mathbf{x}_{2}\right)$ \\
\hline $\mathbf{r}^{2} \mathbf{y} x$ & 0.964 & 0.644 & 0.209 & 0.122 & 0.995 & 0.357 \\
\hline $\mathbf{r y x}$ & $0.982 * *$ & $0.802 * *$ & $0.457 *$ & 0.350 & $0.998 * *$ & $0.597 * *$ \\
\hline $\mathbf{r}^{2} \mathbf{x}_{1} \mathbf{x}_{2}$ & & & & & & \\
\hline $\mathbf{r} \mathbf{x}_{1} \mathbf{x}_{2}$ & & $6 * *$ & & & & \\
\hline Partial & $\left(\mathbf{x}_{1}\right)$ & $\left(\mathbf{x}_{2}\right)$ & $\left(\mathbf{x}_{1}\right)$ & $\left(\mathbf{x}_{2}\right)$ & $\left(x_{1}\right)$ & $\left(\mathbf{x}_{2}\right)$ \\
\hline $\mathbf{r}^{2} \mathbf{y} x_{1} \cdot x_{2}$ & 0.901 & & 0.155 & & 0.994 & \\
\hline $\mathbf{r} \mathbf{y} x_{1} \cdot x_{2}$ & $0.949 * *$ & & 0.394 & & $0.997 * *$ & \\
\hline $\mathbf{r}^{2} \mathbf{y} \mathbf{x}_{2} \cdot \mathbf{x}_{1}$ & & 0.033 & & 0.063 & & 0.126 \\
\hline $\mathbf{r} \mathbf{y} x_{2} \cdot x_{1}$ & & 0.181 & & 0.251 & & 0.355 \\
\hline Multiple & $\left(\mathbf{x}_{1}\right)$ & $\left(\mathbf{x}_{2}\right)$ & $\left(\mathbf{x}_{1}\right)$ & $\left(\mathbf{x}_{2}\right)$ & $\left(\mathbf{x}_{1}\right)$ & $\left(\mathbf{x}_{2}\right)$ \\
\hline $\mathbf{R}^{2} \mathbf{y} \cdot \mathrm{x}_{1} \mathrm{x}_{2}$ & & & & & & \\
\hline Ry. $x_{1} x_{2}$ & & $32 * *$ & & & & \\
\hline & & & groups in bot & ations & & \\
\hline Correlations & Dry weight & Lint weight & & Seeds weight & No. Seeds & \\
\hline Simple & $\left(\mathbf{x}_{1}\right)$ & $\left(\mathbf{x}_{2}\right)$ & & $\left(\mathbf{x}_{1}\right)$ & $\left(\mathbf{x}_{2}\right)$ & \\
\hline $\mathbf{r}^{2} \mathbf{y} x$ & 0.299 & 0.925 & & 0.968 & 0.440 & \\
\hline $\mathbf{r} \mathbf{y} x$ & $0.546 * *$ & $0.962 * *$ & & $0.984 * *$ & $0.664 * *$ & \\
\hline $\mathbf{r}^{2} \mathbf{x}_{1} \mathbf{x}_{2}$ & & & & & & \\
\hline $\mathbf{r} \mathbf{x}_{1} \mathbf{x}_{2}$ & & $35 * *$ & & & & \\
\hline Partial & $\left(\mathbf{x}_{1}\right)$ & $\left(\mathbf{x}_{2}\right)$ & & $\left(\mathbf{x}_{1}\right)$ & $\left(\mathbf{x}_{2}\right)$ & \\
\hline $\mathbf{r}^{2} \mathbf{y} x_{1} \cdot x_{2}$ & 0.019 & & & 0.957 & & \\
\hline $\mathbf{r} \mathbf{y} \mathbf{x}_{1} \cdot \mathbf{x}_{2}$ & 0.139 & & & $0.979 * *$ & & \\
\hline $\mathbf{r}^{2} \mathbf{y} \mathbf{x}_{2} \cdot \mathbf{x}_{1}$ & & 0.896 & & & 0.260 & \\
\hline $\mathbf{r} \mathbf{y} x_{2} \cdot x_{1}$ & & $0.946 * *$ & & & $0.510 * *$ & \\
\hline Multiple & $\left(\mathrm{x}_{1}\right)$ & $\left(\mathbf{x}_{2}\right)$ & & $\left(\mathbf{x}_{1}\right)$ & $\left(\mathbf{x}_{2}\right)$ & \\
\hline $\begin{array}{l}R^{2} y \cdot x_{1} x_{2} \\
R y \cdot x_{1} x_{2}\end{array}$ & & & & & & \\
\hline
\end{tabular}


Table (5): Cont.II

\begin{tabular}{|c|c|c|c|c|c|c|}
\hline \multicolumn{7}{|c|}{$($ G83 x (G75 x 5844)) x G80 } \\
\hline \multirow[b]{3}{*}{$\begin{array}{c}\text { Correlations } \\
\text { Simple } \\
\end{array}$} & \multicolumn{6}{|c|}{ First group in individual locations } \\
\hline & \multicolumn{2}{|c|}{ Beni Souif } & \multicolumn{2}{|c|}{ Minia } & \multicolumn{2}{|c|}{ Assuit } \\
\hline & $\begin{array}{c}\text { Dry weight } \\
\left(\mathbf{x}_{1}\right) \\
\end{array}$ & $\begin{array}{c}\text { Lint weight } \\
\left(\mathbf{x}_{2}\right) \\
\end{array}$ & $\begin{array}{c}\text { Dry weight } \\
\left(\mathrm{x}_{1}\right) \\
\end{array}$ & $\begin{array}{c}\text { Lint weight } \\
\left(\mathbf{x}_{2}\right)\end{array}$ & $\begin{array}{c}\text { Dry weight } \\
\left(\mathrm{x}_{1}\right)\end{array}$ & $\begin{array}{c}\text { Lint weight } \\
\left(\mathrm{x}_{2}\right)\end{array}$ \\
\hline $\begin{array}{l}\mathbf{r}^{2} \mathbf{y} x \\
\mathbf{r} \mathbf{y} x\end{array}$ & $\begin{array}{l}0.173 \\
0.416^{*}\end{array}$ & $\begin{array}{l}0.938 \\
0.968^{* *}\end{array}$ & $\begin{array}{l}0.068 \\
0.261\end{array}$ & $\begin{array}{l}0.209 \\
0.457 *\end{array}$ & $\begin{array}{l}0.193 \\
0.439\end{array}$ & $\begin{array}{l}0.946 \\
0.973 * *\end{array}$ \\
\hline $\begin{array}{l}\mathbf{r}^{2} \mathbf{x}_{1} \mathbf{x}_{2} \\
\mathbf{r} \mathbf{x}_{1} \mathbf{x}_{2} \\
\end{array}$ & \multicolumn{2}{|c|}{$\begin{array}{l}0.184 \\
0.429 *\end{array}$} & \multicolumn{2}{|c|}{$\begin{array}{l}0.098 \\
0.313\end{array}$} & \multicolumn{2}{|c|}{$\begin{array}{l}0.148 \\
0.385 \\
\end{array}$} \\
\hline Partial & $\left(\mathbf{x}_{1}\right)$ & $\left(\mathbf{x}_{2}\right)$ & $\left(\mathbf{x}_{1}\right)$ & $\left(\mathbf{x}_{2}\right)$ & $\left(\mathbf{x}_{1}\right)$ & $\left(\mathbf{x}_{2}\right)$ \\
\hline 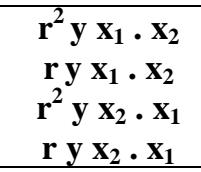 & $\begin{array}{c}0.00002 \\
0.005\end{array}$ & $\begin{array}{l}0.925 \\
0.962 * *\end{array}$ & $\begin{array}{l}0.019 \\
0.139\end{array}$ & $\begin{array}{l}0.168 \\
0.410 \\
\end{array}$ & $\begin{array}{l}0.091 \\
0.302\end{array}$ & $\begin{array}{l}0.939 \\
0.969 * *\end{array}$ \\
\hline Multiple & $\left(\mathbf{x}_{1}\right)$ & $\left(\mathbf{x}_{2}\right)$ & $\left(\mathbf{x}_{1}\right)$ & $\left(\mathbf{x}_{2}\right)$ & $\left(\mathbf{x}_{1}\right)$ & $\left(\mathbf{x}_{2}\right)$ \\
\hline $\begin{array}{l}R^{2} y \cdot x_{1} x_{2} \\
R y \cdot x_{1} x_{2} \\
\end{array}$ & \multicolumn{2}{|c|}{$\begin{array}{l}0.938 \\
0.968 * *\end{array}$} & \multicolumn{2}{|c|}{$\begin{array}{l}0.224 \\
0.474 * \\
\end{array}$} & \multicolumn{2}{|c|}{$\begin{array}{l}0.951 \\
0.975^{* *}\end{array}$} \\
\hline \multicolumn{7}{|c|}{ Second group in individual locations } \\
\hline & \multicolumn{2}{|c|}{ Beni Souif } & \multicolumn{2}{|c|}{ Minia } & \multicolumn{2}{|c|}{ Assuit } \\
\hline Correlations & $\begin{array}{c}\text { Seeds } \\
\text { weight } \\
\left(\mathrm{x}_{1}\right)\end{array}$ & $\begin{array}{c}\text { No. Seeds } \\
\left(\mathbf{x}_{2}\right) \\
\end{array}$ & $\begin{array}{c}\text { Seeds weight } \\
\left(\mathrm{x}_{1}\right) \\
\end{array}$ & $\begin{array}{c}\text { No. Seeds } \\
\left(\mathbf{x}_{2}\right)\end{array}$ & $\begin{array}{c}\text { Seeds weight } \\
\left(\mathrm{x}_{1}\right) \\
\end{array}$ & $\begin{array}{c}\text { No. Seeds } \\
\left(\mathbf{x}_{2}\right) \\
\end{array}$ \\
\hline $\mathbf{r}^{2} \mathbf{y} x$ & $\begin{array}{l}0.973 \\
0.987 * *\end{array}$ & $\begin{array}{l}0.542 \\
0.737 * *\end{array}$ & $\begin{array}{l}0.232 \\
0.482 *\end{array}$ & $\begin{array}{l}0.136 \\
0.368\end{array}$ & $\begin{array}{l}0.981 \\
0.990 * *\end{array}$ & $\begin{array}{l}0.160 \\
0.400\end{array}$ \\
\hline $\begin{array}{l}\mathbf{r}^{2} x_{1} x_{2} \\
r x_{1} x_{2}\end{array}$ & \multicolumn{2}{|c|}{$\begin{array}{l}0.532 \\
0.730 * *\end{array}$} & \multicolumn{2}{|c|}{$\begin{array}{l}0.078 \\
0.279\end{array}$} & \multicolumn{2}{|c|}{$\begin{array}{l}0.137 \\
0.370\end{array}$} \\
\hline Partial & $\left(\mathbf{x}_{1}\right)$ & $\left(\mathbf{x}_{2}\right)$ & $\left(\mathbf{x}_{1}\right)$ & $\left(\mathbf{x}_{2}\right)$ & $\left(\mathbf{x}_{1}\right)$ & $\left(\mathbf{x}_{2}\right)$ \\
\hline $\begin{array}{l}\mathbf{r}^{2} \mathbf{y} x_{1} \cdot x_{2} \\
\mathbf{r} \mathbf{y}_{1} \cdot x_{2} \\
\mathbf{r}^{2} \mathbf{y x}_{2} \cdot \mathbf{x}_{1} \\
\mathbf{r} \mathbf{y} x_{2} \cdot x_{1}\end{array}$ & $\begin{array}{l}0.943 \\
0.971 * *\end{array}$ & $\begin{array}{l}0.022 \\
0.149\end{array}$ & $\begin{array}{l}0.180 \\
0.425\end{array}$ & $\begin{array}{l}0.077 \\
0.278\end{array}$ & $\begin{array}{l}0.979 \\
0.989 * *\end{array}$ & $\begin{array}{l}0.067 \\
0.259 \\
\end{array}$ \\
\hline Multiple & $\left(\mathbf{x}_{1}\right)$ & $\left(\mathbf{x}_{2}\right)$ & $\left(\mathbf{x}_{1}\right)$ & $\left(\mathbf{x}_{2}\right)$ & $\left(\mathbf{x}_{1}\right)$ & $\left(\mathbf{x}_{2}\right)$ \\
\hline $\begin{array}{l}R^{2} y \cdot x_{1} x_{2} \\
R y \cdot x_{1} x_{2} \\
\end{array}$ & \multicolumn{2}{|c|}{$\begin{array}{l}0.974 \\
0.987 * *\end{array}$} & & & & $\begin{array}{l}2 \\
1 * * \\
\end{array}$ \\
\hline & & & roups in bot & cations & & \\
\hline Correlations & Dry weight & Lint weight & & Seeds weight & No. Seeds & \\
\hline Simple & $\left(\mathbf{x}_{1}\right)$ & $\left(\mathbf{x}_{2}\right)$ & & $\left(\mathbf{x}_{1}\right)$ & $\left(\mathbf{x}_{2}\right)$ & \\
\hline $\begin{array}{l}\mathbf{r}^{2} \mathbf{y} x \\
\mathbf{r} \mathbf{y} x\end{array}$ & $\begin{array}{l}0.223 \\
0.472 * *\end{array}$ & $\begin{array}{l}0.909 \\
0.954 * *\end{array}$ & & $\begin{array}{l}0.963 \\
0.981 * *\end{array}$ & $\begin{array}{l}0.346 \\
0.588 * *\end{array}$ & \\
\hline $\begin{array}{l}\mathbf{r}^{2} \mathbf{x}_{1} \mathbf{x}_{2} \\
\mathbf{r} \mathbf{x}_{1} \mathbf{x}_{2}\end{array}$ & $\begin{array}{r}0 . \\
0 .\end{array}$ & $9 * *$ & & & $\begin{array}{l}6 \\
6 * * \\
\end{array}$ & \\
\hline Partial & $\left(\mathbf{x}_{1}\right)$ & $\left(\mathbf{x}_{2}\right)$ & & $\left(\mathbf{x}_{1}\right)$ & $\left(\mathbf{x}_{2}\right)$ & \\
\hline $\begin{array}{l}\mathbf{r}^{2} \mathbf{y ~ x}_{1} \cdot \mathbf{x}_{2} \\
\mathbf{r} \mathbf{y}_{1} \cdot \mathbf{x}_{2} \\
\mathbf{r}^{2} \mathbf{y ~ x}_{2} \cdot \mathbf{x}_{1} \\
\mathbf{r} \mathbf{y} x_{2} \cdot x_{1}\end{array}$ & $\begin{array}{l}0.003 \\
0.057\end{array}$ & $\begin{array}{l}0.884 \\
0.940 * *\end{array}$ & & $\begin{array}{l}0.960 \\
0.980 * *\end{array}$ & $\begin{array}{l}0.299 \\
0.547 * *\end{array}$ & \\
\hline Multiple & $\left(\mathbf{x}_{1}\right)$ & $\left(\mathbf{x}_{2}\right)$ & & $\left(\mathbf{x}_{1}\right)$ & $\left(\mathbf{x}_{2}\right)$ & \\
\hline $\begin{array}{l}R^{2} y \cdot x_{1} x_{2} \\
R y \cdot x_{1} x_{2} \\
\end{array}$ & & & & & & \\
\hline
\end{tabular}


Table (5): Cont.III

\begin{tabular}{|c|c|c|c|c|c|c|}
\hline \multicolumn{7}{|c|}{ G90 x Australian } \\
\hline \multirow[b]{3}{*}{ Correlations } & \multicolumn{6}{|c|}{ First group in individual locations } \\
\hline & \multicolumn{2}{|c|}{ Beni Souif } & \multicolumn{2}{|c|}{ Minia } & \multicolumn{2}{|c|}{ Assuit } \\
\hline & \multirow{2}{*}{$\begin{array}{c}\text { Dry weight } \\
\left(\mathrm{x}_{1}\right)\end{array}$} & \multirow{2}{*}{$\begin{array}{c}\text { Lint weight } \\
\left(\mathbf{x}_{2}\right)\end{array}$} & \multirow{2}{*}{$\begin{array}{c}\text { Dry weight } \\
\left(\mathbf{x}_{1}\right)\end{array}$} & \multirow{2}{*}{$\begin{array}{c}\text { Lint weight } \\
\left(\mathbf{x}_{2}\right)\end{array}$} & \multirow{2}{*}{$\begin{array}{c}\text { Dry weight } \\
\left(\mathrm{x}_{1}\right)\end{array}$} & \multirow{2}{*}{$\begin{array}{c}\text { Lint weight } \\
\left(\mathrm{x}_{2}\right)\end{array}$} \\
\hline Simple & & & & & & \\
\hline $\mathbf{r}^{2} \mathbf{y} \mathbf{x}$ & 0.222 & 0.940 & 0.061 & 0.225 & 0.001 & 0.992 \\
\hline $\mathbf{r} \mathbf{y} \mathbf{x}$ & $0.471 *$ & $0.969 * *$ & 0.247 & $0.474 *$ & 0.029 & $0.996 * *$ \\
\hline $\mathbf{r}^{2} \mathbf{x}_{1} \mathbf{x}_{2}$ & \multirow{2}{*}{\multicolumn{2}{|c|}{$\begin{array}{l}0.205 \\
0.453^{*} \\
\end{array}$}} & \multirow{2}{*}{\multicolumn{2}{|c|}{$\begin{array}{l}0.085 \\
0.292 \\
\end{array}$}} & \multirow{2}{*}{\multicolumn{2}{|c|}{$\begin{array}{l}0.002 \\
0.044\end{array}$}} \\
\hline $\mathbf{r} \mathbf{x}_{1} \mathbf{x}_{2}$ & & & & & & \\
\hline Partial & $\left(\mathbf{x}_{1}\right)$ & $\left(\mathbf{x}_{2}\right)$ & $\left(\mathbf{x}_{1}\right)$ & $\left(\mathbf{x}_{2}\right)$ & $\left(\mathbf{x}_{1}\right)$ & $\left(\mathbf{x}_{2}\right)$ \\
\hline $\mathbf{r}^{2} \mathbf{y ~ x}_{1} \cdot \mathbf{x}_{2}$ & \multirow{4}{*}{$\begin{array}{l}0.021 \\
0.146\end{array}$} & & \multirow{4}{*}{$\begin{array}{l}0.017 \\
0.129\end{array}$} & & \multirow{4}{*}{$\begin{array}{l}0.030 \\
0.172\end{array}$} & \\
\hline $\mathbf{r} \mathbf{y} x_{1} \cdot \mathbf{x}_{2}$ & & & & & & \\
\hline $\mathbf{r}^{2} \mathbf{y} \mathbf{x}_{2} \cdot \mathbf{x}_{1}$ & & 0.924 & & 0.188 & & 0.992 \\
\hline $\mathbf{r} \mathbf{y x}_{2} \cdot \mathbf{x}_{1}$ & & $0.961 * *$ & & 0.434 & & $0.996 * *$ \\
\hline Multiple & $\left(\mathbf{x}_{1}\right)$ & $\left(\mathbf{x}_{2}\right)$ & $\left(\mathbf{x}_{1}\right)$ & $\left(\mathbf{x}_{2}\right)$ & $\left(\mathbf{x}_{1}\right)$ & $\left(\mathbf{x}_{2}\right)$ \\
\hline $\mathbf{R}^{2} \mathbf{y} \cdot \mathbf{x}_{1} \mathbf{x}_{2}$ & \multirow{2}{*}{\multicolumn{2}{|c|}{$\begin{array}{l}0.941 \\
0.970 * *\end{array}$}} & \multirow{2}{*}{\multicolumn{2}{|c|}{$\begin{array}{l}0.238 \\
0.488 *\end{array}$}} & \multirow{2}{*}{\multicolumn{2}{|c|}{$\begin{array}{l}0.992 \\
0.996^{* *}\end{array}$}} \\
\hline Ry. $x_{1} x_{2}$ & & & & & & \\
\hline \multicolumn{7}{|c|}{ Second group in individual locations } \\
\hline & Ben & Souif & & & & \\
\hline Correlations & Seeds & No. Seeds & Seeds weight & No. Seeds & Seeds weight & No. Seeds \\
\hline Simple & $\left(\mathbf{x}_{1}\right)$ & $\left(\mathbf{x}_{2}\right)$ & $\left(\mathbf{x}_{1}\right)$ & $\left(\mathbf{x}_{2}\right)$ & $\left(\mathbf{x}_{1}\right)$ & $\left(\mathbf{x}_{2}\right)$ \\
\hline $\mathbf{r}^{2} \mathbf{y} x$ & 0.975 & 0.559 & 0.241 & 0.113 & 0.995 & 0.494 \\
\hline $\mathbf{r} \mathbf{y} x$ & $0.987 * *$ & $0.748 * *$ & $0.491 *$ & 0.336 & $0.997 * *$ & $0.703 * *$ \\
\hline $\mathbf{r}^{2} \mathbf{x}_{1} \mathbf{x}_{2}$ & & & & & & \\
\hline $\mathbf{r} \mathbf{x}_{1} \mathbf{x}_{2}$ & & $96 * *$ & & & & $5 * *$ \\
\hline Partial & $\left(\mathbf{x}_{1}\right)$ & $\left(\mathbf{x}_{2}\right)$ & $\left(\mathbf{x}_{1}\right)$ & $\left(\mathbf{x}_{2}\right)$ & $\left(\mathbf{x}_{1}\right)$ & $\left(\mathbf{x}_{2}\right)$ \\
\hline $\mathbf{r}^{2} \mathbf{y} \mathbf{x}_{1} \cdot \mathbf{x}_{2}$ & 0.959 & & 0.183 & & 0.990 & \\
\hline $\mathbf{r} \mathbf{y} \mathbf{x}_{1} \cdot \mathbf{x}_{2}$ & $0.979 * *$ & & 0.427 & & $0.995 * *$ & \\
\hline $\mathbf{r}^{2} \mathbf{y} \mathbf{x}_{2} \cdot \mathbf{x}_{1}$ & & 0.282 & & 0.045 & & 0.036 \\
\hline $\mathbf{r} \mathbf{y} x_{2} \cdot x_{1}$ & & $0.531 *$ & & 0.211 & & 0.190 \\
\hline Multiple & $\left(\mathbf{x}_{1}\right)$ & $\left(\mathbf{x}_{2}\right)$ & $\left(\mathbf{x}_{1}\right)$ & $\left(\mathbf{x}_{2}\right)$ & $\left(x_{1}\right)$ & $\left(\mathbf{x}_{2}\right)$ \\
\hline$R^{2} y \cdot x_{1} x_{2}$ & & & & & & \\
\hline$R y . x_{1} x_{2}$ & & $91 * *$ & & & & $8 * *$ \\
\hline & & Two & roups in both & ations & & \\
\hline Correlations & Dry weight & Lint weight & & Seeds weight & No. Seeds & \\
\hline Simple & $\left(\mathbf{x}_{1}\right)$ & $\left(\mathbf{x}_{2}\right)$ & & $\left(\mathbf{x}_{1}\right)$ & $\left(\mathbf{x}_{2}\right)$ & \\
\hline $\mathbf{r}^{2} \mathbf{y x}$ & 0.030 & 0.953 & & 0.980 & 0.451 & \\
\hline $\mathbf{r} \mathbf{y} x$ & 0.172 & $0.976 * *$ & & $0.990 * *$ & $0.672 * *$ & \\
\hline $\mathbf{r}^{2} \mathbf{x}_{1} \mathbf{x}_{2}$ & & & & & & \\
\hline $\mathbf{r} \mathbf{x}_{1} \mathbf{x}_{2}$ & & & & & $0 * *$ & \\
\hline Partial & $\left(\mathbf{x}_{1}\right)$ & $\left(\mathbf{x}_{2}\right)$ & & $\left(\mathbf{x}_{1}\right)$ & $\left(\mathbf{x}_{2}\right)$ & \\
\hline $\mathbf{r}^{2} \mathrm{y} \mathrm{x}_{1} \cdot \mathrm{x}_{2}$ & 0.0004 & & & 0.970 & & \\
\hline $\mathbf{r} \mathbf{y} \mathbf{x}_{1} \cdot \mathbf{x}_{2}$ & 0.020 & & & $0.985 * *$ & & \\
\hline $\mathbf{r}^{2} \mathbf{y} \mathbf{x}_{2} \cdot \mathbf{x}_{1}$ & & 0.952 & & & 0.191 & \\
\hline $\mathbf{r} \mathbf{y} x_{2} \cdot x_{1}$ & & $0.976 * *$ & & & $0.436 * *$ & \\
\hline Multiple & $\left(\mathbf{x}_{1}\right)$ & $\left(\mathbf{x}_{2}\right)$ & & $\left(\mathbf{x}_{1}\right)$ & $\left(\mathbf{x}_{2}\right)$ & \\
\hline $\begin{array}{l}R^{2} y \cdot x_{1} x_{2} \\
R y \cdot x_{1} x_{2}\end{array}$ & & $\begin{array}{l}53 \\
76 * *\end{array}$ & & & 34 & \\
\hline
\end{tabular}

*, ** Significant at 0.05 and 0.01 levels, respectively. 
the variability in yield, $\left(100 \mathrm{x} \mathrm{r}^{2} \mathrm{y} \mathrm{x}_{1}\right)$ of G80, G90, (G83 x (G75 x 5844)) x G80 and G90 x Australian, respectively. On the other hand, lint cotton weight alone accounted for $94.3 \%, 92.5 \%$, $90.9 \%$ and $95.3 \%$ of the variability in yield, (100 $\mathrm{x} \mathrm{r}^{2} \mathrm{y}_{2}$ ) for the same order of genotypes. Both dry and lint weight jointly accounted for $94.4 \%$, $92.7 \%, 91 \%$ and $95.3 \%$ of the variability in yield, $\left(100 \times R^{2} y \cdot x_{1} x_{2}\right)$ for the same order of genotypes.

Concerning the second group, seeds weight and the number of seeds per boll were significantly positively simply correlated with yield for all genotypes.

Seeds weight per boll showed significant positive partial correlation with yield when the number of seeds per boll is held constant for all genotypes. Also, the number of seeds per boll was significantly positively partially correlated with yield when seeds weight per boll is held constant for all genotypes.

Both seeds weight and the number of seeds per boll showed significant positive multiple correlation with yield for all genotypes.

The results of the second group revealed that seeds weight per boll alone accounted for $98 \%$, $96.8 \%, 96.3 \%$ and $98 \%$ of the variability in yield, (100 x r $\left.\mathrm{r}^{2} \mathrm{y} \mathrm{x}_{1}\right)$ of G80, G90, (G83 x (G75 x 5844)) x G80 and G90 x Australian, respectively. On the other hand, the number of seeds per boll alone accounted for $43.4 \%, 44 \%, 34.6 \%$ and $45.1 \%$ of the variability in yield, $\left(100 \mathrm{x} \mathrm{r}^{2} \mathrm{y} \mathrm{x}_{2}\right)$ for the same order of genotypes. Both seeds weight and the number of seeds per boll jointly accounted for $98.3 \%, 97.6 \%, 97.4 \%$ and $98.4 \%$ of the variability in yield, $\left(100 \times \mathrm{R}^{2} \mathrm{y} \cdot \mathrm{x}_{1} \mathrm{x}_{2}\right)$ for the same order of genotypes.

For the explanation of such results, a perfect correlation would be extremely rare in biological material though values above -0.9 and 0.9 are not uncommon. It is difficult to give a clear interpretation of different values of the correlation coefficient, but values above -0.5 or 0.5 are considered to indicate a close relationship; those between -0.3 and -0.50 (or 0.3 and 0.5 ), moderately close; and those below -0.3 or 0.3 , little or no relationship. It is sometimes stated that the quantitative relationship between the two variables is given by the square of the correlation coefficient, if 1 gives complete interdependence. In other words, differences in the size of the correlation at higher values for $r$ have more meaning than similar differences for low values.

Just as $r^{2}$ was called the coefficient of determination, $\mathrm{R}^{2}$ is called the multiple coefficient of determination. It is the proportion of the variation in $y$ accounted for by the variation in the two or more independent variables.

The multiple coefficient of correlation, R, shows how closely the points in the ellipsoid are clustered around the regression plane. The value of $\mathrm{R}$ ranging from zero to one. Furthermore, it is always at least as large as the largest simple and partial coefficients. This fact serves as a good check on the calculations.

\section{REFERENCES}

Fowler, J., Cohen L. and Jarvis, P. (1998). Practical statistics for field biology. Second Edit, John Wiley and Sons, New York, U.S.A.

Gill S. S. (1981). Correlation and path coefficient analysis of yield with yield components in Upland cotton. Crop Improvement. $8: 23$ 27.

Idris H. A.(2002). Models for evaluating Egyptian cotton responses to environmental variables. Ph.D. Thesis, Fac., of Agric., Cairo Univ., Egypt.

Idris H. A., Baker Kh. M. and Mahrous H. (2011). Evaluating some Egyptian cotton genotypes over two stages. Bull. Fac. Agric. Cairo Univ., 62 (4): 395- 408.

Little T.M. and Hills F. J. (1978). Agricultural Experimentation Design and Analysis. John Wiley and Sons, New York., U.S.A.

Mahrous H., El Fisheikawy A. B, Baker K. M. and Idris H. A. (2012). Correlation and path coefficient analysis for yield and its components in Egyptian cotton. Minia J. Agric. Res. 32 (5) 49- 57.

Patil M. S. and Mensinkai S. W. (1972). Selection index for yield in diploid cotton $(G$. herbaceum L.). Madras Agric. J. 59 : 660. Plant Breed. Abst, 43, 8003).

Roger G. P. (1994). Agricultural Field Experiments Design and Analysis. Marcel Dekker, Inc. New York, U.S.A. 


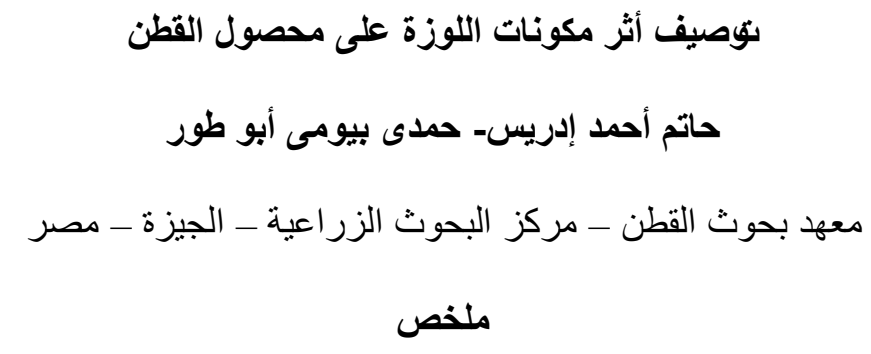

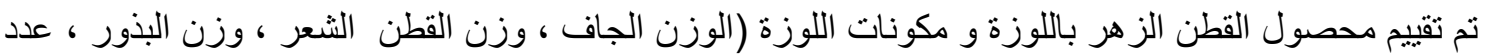

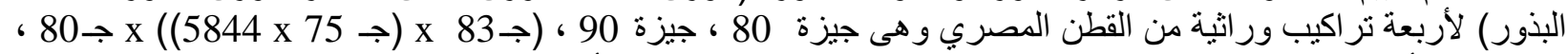

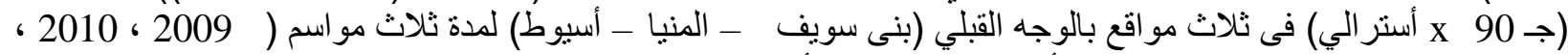

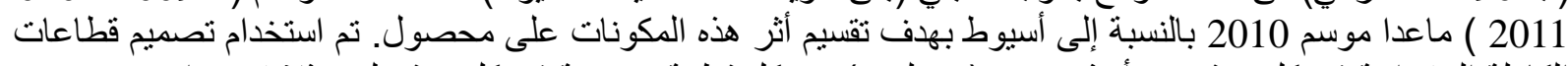

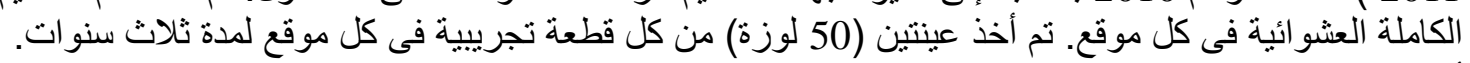

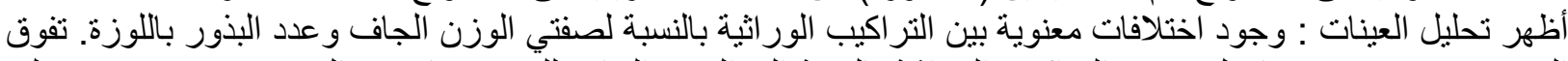

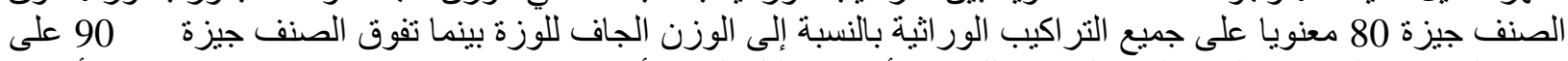

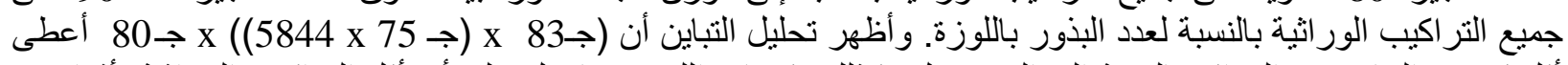

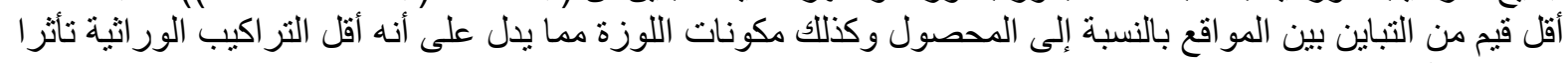

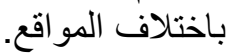

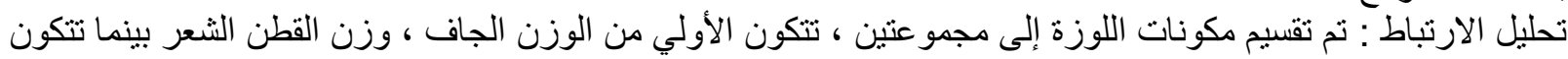

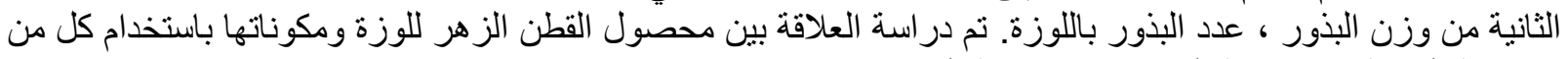

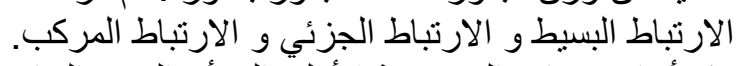

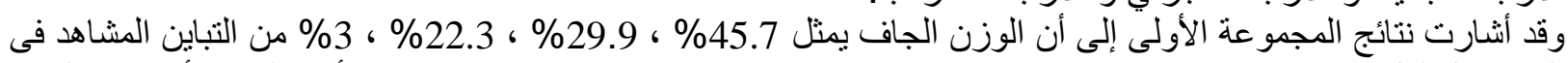

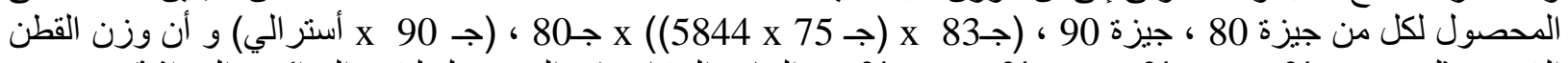

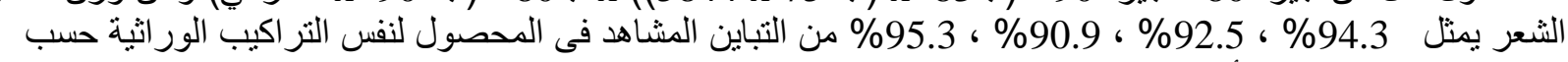

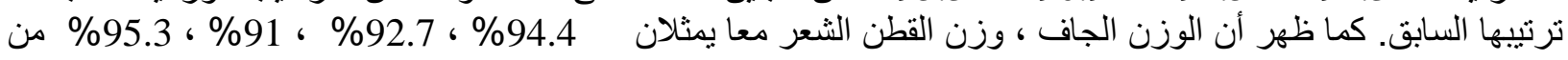

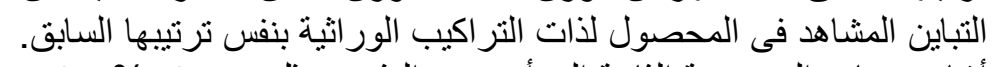

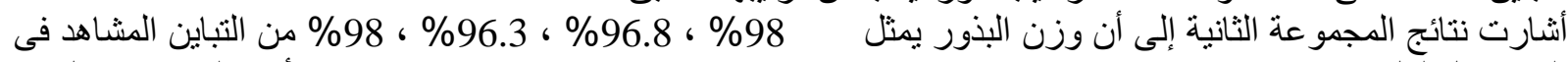

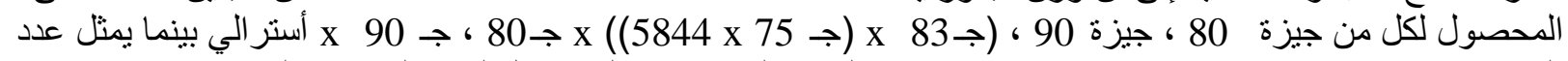

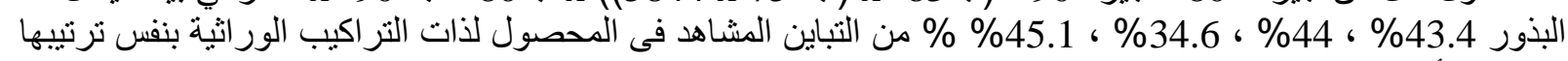

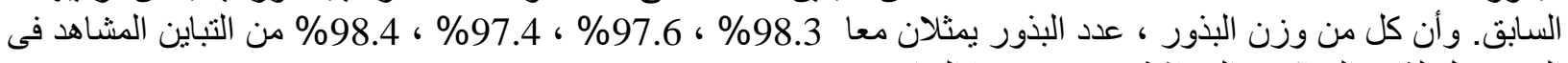

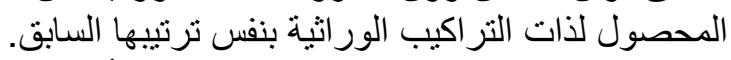

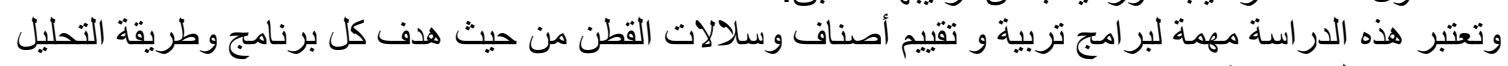

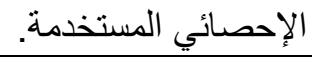
المجلة العلمية لكلية الزراعة - جامعة القاهرة - المجلد (64) العدد الثانى (أبريل2013):129-139. 